\title{
Incidência e Fatores de Risco para Hemorragia Pulmonar em Recém-Nascidos de Muito Baixo Peso
}

\author{
Incidence and Risk Factors for Pulmonary Hemorrhage in \\ Very Low Weight Newborns
}

\author{
Cintia Suemy Uehara ${ }^{1}$ \\ Ana Claudia Moraes Medeiros de Lima² \\ Arthur Pedro Marinho ${ }^{2}$ \\ Lorena de Carvalho Monte de Prada ${ }^{2}$ \\ Kerolaynne Fonseca de Lima ${ }^{3}$ \\ Suianny Karla de Oliveira Macedo ${ }^{2}$ \\ Camila Dayze Pereira Santos ${ }^{3}$ \\ Ana Verônica Dantas de Carvalho ${ }^{4}$ \\ Wilton Rodrigues Medeiros ${ }^{5}$ \\ Anna Christina do Nascimento Granjeiro Barreto ${ }^{6}$
}

RESUMO

Objetivo: Determinar a incidência de hemorragia pulmonar (HP) e os fatores de risco associados em prematuros com idade gestacional $(\mathrm{IG}) \leq 32$ semanas e de muito baixo peso. Metodologia: Estudo longitudinal, observacional, prospectivo com recém-nascidos $\leq 32$ semanas e de muito baixo peso, internados em Unidade de Terapia Intensiva Neonatal em Natal/RN, no período de outubro/2017 a setembro/2018. A incidência de HP foi determinada e as variáveis obstétricas e do período neonatal foram avaliadas como fatores de risco. A análise estatística realizada foi descritiva e inferencial com a utilização dos Testes Qui-quadrado, t de Student e regressão logística multivariada. Resultados: Foram incluídos no estudo 123 prematuros, dos quais $37(30,1 \%)$ apresentaram HP. As médias de IG e peso de nascimento dos bebês que apresentaram essa complicação foram 27,2 semanas, e $875,5 \mathrm{~g}$, respectivamente. As variáveis, menor IG e peso ao nascimento, o não uso de corticoide antenatal, necessidade de manobras de reanimação na sala de parto, Apgar no $5^{\circ}$ minuto $<7$, temperatura à admissão, administração de surfactante, persistência do canal arterial e sepse precoce foram identificadas como fatores de risco para HP. Na regressão logística os fatores que permaneceram associados à HP foram o não uso de corticoide antenatal, peso ao nascimento e persistência do canal arterial. Conclusão: A hemorragia pulmonar é um evento clínico com importante incidência em recém-nascidos prematuros e de muito baixo peso, com fatores de risco associados à prematuridade e assistência inadequada a gestante e ao recém-nascido, sendo de extrema importância o cuidado perinatal para evitar este desfecho que confere maior morbimortalidade aos pacientes.

\section{DESCRITORES}

Prematuridade. Recém-Nascido de Muito Baixo Peso. Corticoide Antenatal. Hemorragia Pulmonar. Persistência do Canal Arterial.

\begin{abstract}
Objective: To determine the incidence of pulmonary hemorrhage $(\mathrm{PH})$ and evaluate the associated risk factors in neonates with $\leq 32$ weeks of gestational age and very low birthweight. Methodology: This is a longitudinal, observational and prospective study in which we analyzed data from neonates with $\leq 32$ weeks gestational age and very low birth weight admitted to a Neonatal Intensive Care Unit in Natal/RN, during October 2017 and September 2018. We have determined the pulmonary hemorrhage incidence and have analyzed the associated risk factors during pregnancy and neonatal period. The statistical analysis was descriptive and inferential using the Chi-square test, Student's T test and the multivariate logistic regression model. Results: 123 premature infants were included in the study and 37 of them $(30,1 \%)$ have developed $\mathrm{PH}$. For those babies with $\mathrm{PH}$ the mean gestational age was 27.2 weeks and the mean birth weight was $875.5 \mathrm{~g}$. We found out to be important $\mathrm{PH}$ risk factors: lower gestational age and birthweight, absence of antenatal corticosteroid therapy, need for delivery room resuscitation, Apgar score in the $5^{\text {th }}$ minute $<7$, admission temperature, use of surfactant therapy, persistent ductus arteriosus (PDA) and early onset sepsis. Only the absence of antenatal corticosteroid therapy, low birthweight and PDA remained significant after the multivariate logistic regression analysis. Conclusion: $\mathrm{PH}$ is a high incidence clinic event in premature infants and very low birth weight neonates. The risk factors are associated with prematurity itself and inadequate assistance to pregnancy and premature labor, being extremely important to focus on a good perinatal care to avoid the development of such high morbidity and mortality disease.
\end{abstract}

\section{DESCRIPTORS}

Prematurity. Very Low Birth Weight Newborns. Antenatal Corticosteroid. Pulmonary Hemorrhage. Persistent Ductus Arteriosus.

${ }^{1}$ Aluna do Programa de Residência Médica em Neonatologia na Maternidade Escola Januário Cicco (MEJC), Universidade Federal do Rio Grande do Norte (UFRN), Natal, Rio Grande do Norte, Brasil.

2 Médico(a) pediatra. Aluno(a) do Programa de Residência Médica em Neonatologia na Maternidade Escola Januário Cicco (MEJC), Universidade Federal do Rio Grande do Norte (UFRN), Natal, Rio Grande do Norte, Brasil.

${ }^{3}$ Nutricionista. Aluna do Programa de Residência Integrada Multiprofissional em Saúde na Maternidade Escola Januário Cicco (MEJC), Universidade Federal do Rio Grande do Norte (UFRN), Natal, Rio Grande do Norte, Brasil.

${ }^{4}$ Nutricionista. Maternidade Escola Januário Cicco (MEJC), Universidade Federal do Rio Grande do Norte (UFRN), Natal, Rio Grande do Norte, Brasil.

${ }^{5}$ Sanitarista. Hospital Universitário Ana Bezerra (HUAB), Universidade Federal do Rio Grande do Norte (UFRN), Natal, Rio Grande do Norte, Brasil.

${ }^{6}$ Maternidade Escola Januário Cicco (MEJC), Universidade Federal do Rio Grande do Norte (UFRN), Natal, Rio Grande do Norte, Brasil. 
A hemorragia pulmonar (HP) é uma complicação frequente em recém-nascidos prematuros de muito baixo peso, sendo sua incidência inversamente proporcional ao peso e a idade gestacional ao nascimento. Os critérios utilizados para a definição de HP ainda são controversos na literatura, não existindo um consenso entre autores e serviços de unidades de terapia intensiva neonatais (UTIN). Em muitas pesquisas ela é definida como evento clínico onde o recém-nascido ( $R N)$ evolui com a presença de sangue em cânula endotraqueal e existe deterioração clínica, hemodinâmica e/ou necessidade de aumento de parâmetros na ventilação mecânica1. Alguns trabalhos ainda acrescentam definição radiográfica a esses critérios (infiltrados pulmonares novos seguidos ao sangramento $)^{2,3} \mathrm{e}$ queda aguda de hematócrito com valor maior ou igual a $10 \%$ em relação ao que precedeu o sangramento ${ }^{3}$.

A maioria dos estudos sobre HP existentes na literatura foi realizada em centros pequenos ou com poucos pacientes, sendo difícil estimar a real incidência de tal morbidade. Além disso, como já citado, existe uma variação em relação a própria definição dessa complicação. Um grande estudo americano publicado em 2018, incluindo dados de 340 UTIN dos Estados Unidos com RN prematuros que desenvolveram HP nos 7 primeiros dias de vida, encontrou uma incidência que variou de acordo com a idade gestacional de nascimento: 86/1000 admissões em RN com 23 semanas, ocorrendo uma redução gradual conforme o aumento de idade gestacional (IG), chegando a 0,6-1,9/1000 admissões em RN com 32 semanas ou mais de nascimento ${ }^{4}$. Pesquisa brasileira, realizada na Universidade de São Paulo (USP) de Ribeirão Preto em 2014, encontrou uma incidência de HP igual a 6,7/1000 nascimentos, sendo 80/1000 entre os RN de muito baixo peso'.

Segundo dados da literatura, estudos mostram diversos fatores de risco associados a ocorrência de HP, sendo os principais deles: prematuridade $\mathrm{e}^{1,3,4}$, menor peso de nascimento ${ }^{1,3,4}$, presença de canal arterial patente grande ou com repercussão hemodinâmica verificada clinicamente ou ao ecocardiograma ${ }^{2,4-9}$, uso de surfactante por síndrome de desconforto respiratório $0^{3,10,14}$ e ausência de corticoterapia materna antenatal ${ }^{11,12}$. Em alguns estudos a real prevalência e associação com o canal arterial foi difícil de ser definida, pois vários bebês foram a óbito após o sangramento pulmonar antes que um ecocardiograma pudesse ter sido realizado². Outros fatores de risco encontrados foram: uso de hemoderivados previamente a ocorrência de $\mathrm{HP}^{1}$, intubação em sala de parto ${ }^{1} \mathrm{e}$ asfixia neonatal ${ }^{3}$.

A HP consiste ainda em um evento grave, que pode levar a desfechos clínicos negativos e de mau prognóstico, sendo normalmente associada a altas taxas de mortalidade, principalmente em recém-nascidos de menor IG, podendo chegar a $40,6 \%$ na primeira semana de vida e $56,9 \%$ após esse período ${ }^{4}$. No estudo de Ferreira et al. ${ }^{1}$ foi encontrada uma taxa de óbito de $65 \%$ nos pacientes que evoluíram com HP, versus $21 \%$ nos que não tiveram¹. Em outro estudo taiwanês de 2011, com 21 centros de referência, a hemorragia pulmonar foi responsável por $10,5 \%$ das mortes nos bebês prematuros de muito baixo peso ${ }^{13}$. Além disso, a HP também está associada ao desenvolvimento de outras comorbi- 
dades como a hemorragia peri-intraventricular (HPIV) grave (graus 3 e 4) ${ }^{2-4}$, maior tempo de ventilação mecânica ${ }^{1}$, retinopatia da prematuridade (ROP) estágio 2 ou mais ${ }^{15}$, doença pulmonar crônica com um padrão intersticial em evolução na radiografia torácica ${ }^{16} \mathrm{e}$ maior ocorrência de choque 4 .

No Brasil foram realizadas poucas pesquisas para avaliar essa importante morbidade da prematuridade, sendo a maioria delas retrospectivas. Assim, este trabalho tem como objetivo uma melhor definição do perfil de recém-nascidos mais predispostos a desenvolver tal complicação, analisando sua incidência e os principais fatores de risco associados, tornando possível uma melhora no serviço de terapia intensiva com relação à prevenção desse evento clínico, evitando o aumento de morbimortalidade de prematuros e melhorando o prognóstico desses bebês.

\section{METODOLOGIA}

Estudo do tipo observacional, longitudinal e prospectivo, o qual foi realizado em UTIN de uma maternidade escola no Estado do Rio Grande do Norte (Maternidade Escola Januário Cicco - MEJC/UFRN), referência em gestação de alto risco, durante o período de outubro de 2017 a setembro de 2018, com amostra por conveniência. Essa instituição tem cerca de 300 partos mensais, sendo $30 \%$ deles prematuros. Os dados da pesquisa foram extraídos dos prontuários maternos e da criança com posterior preenchimento de questionário elaborado pelos pesquisadores, tendo os recém-nascidos sido acompanhados do momento da admissão na UTIN até o desfecho final (alta hospitalar ou óbito).
O estudo foi aprovado pelo Comitê de Ética em Pesquisa do Hospital Universitário Onofre Lopes (HUOL), conforme Certificado de Apresentação para Apreciação Ética (CAAE) 11307319.3.0000.5292.

Os recém-nascidos incluídos no estudo apresentavam prematuridade $\leq 32$ semanas de IG e peso de nascimento menor de $1500 \mathrm{~g}$. Os critérios de exclusão foram: RNs diagnosticados com infecções congênitas (toxoplasmose, rubéola, citomegalovírus, herpes simples, sífilis e arbovirose), malformações congênitas graves, erros inatos do metabolismo e caso de gemelaridade múltipla, acima de dois fetos.

Para o diagnóstico de HP foram considerados os bebês que apresentaram sangue no tubo orotraqueal associado a necessidade de aumento de parâmetros ventilatórios e/ou piora hemodinâmica. As variáveis quantitativas analisadas como fatores de risco foram: peso ao nascer, IG ao nascimento (estimada por meio da ultrassonografia de primeiro trimestre ou pela data da última menstruação) e temperatura do bebê à admissão em UTIN, obtida com termômetro axilar. Com relação às variáveis categóricas, foram estudadas como fatores de risco para HP as seguintes: dados da gestação como uso de corticoide antenatal (uma ou duas doses); presença de hipertensão gestacional materna, tipo de parto (cesárea ou vaginal); dados do RN como sexo, necessidade de manobras de reanimação em sala de parto; valor de Apgar no $5^{\circ}$ minuto de vida, necessidade de uso de surfactante intratraqueal para tratamento de síndrome do desconforto respiratório, ocorrência de infecções (segundo critérios definidos pela Agência Nacional de Vigilância Sanitá- 
ria - ANVISA), sendo consideradas precoces quando se manifestam nas primeiras 48 horas de vida e tardias quando ocorrem após esse período ${ }^{17}$, presença de persistência do canal arterial (definida como canal arterial patente com fluxo ao ecocardiograma após $72 \mathrm{~h}$ de vida, diagnosticado com o aparelho de Ecocardiograma Vivid I da GE, exame realizado por cardiologistas pediátricos da instituição); classificação do peso para idade gestacional ao nascimento, tendo sido considerados pequenos para idade gestacional (PIG) os RN com peso ao nascimento abaixo do percentil 10 na curva do Projeto Intergrowth ${ }^{18,19}$ e presença de HPIV, com definição através da classificação de Papile et al. ${ }^{20}$, diagnóstico dado pela ultrassonografia transfontanelar, realizada com o equipamento de ultrassonografia Philips Affiniti 50, com transdutor Linear L12-4 (4 - $12 \mathrm{MHz}$ ), por ultrassonografistas da instituição. Além disso, as variáveis peso e idade gestacional ao nascimento também foram analisadas de forma categórica, tendo o peso sido dividido em dois grupos: até $999 \mathrm{~g} \mathrm{e}$ $\geq 1000 \mathrm{~g}$ e a idade gestacional ao nascimento também categorizada em dois grupos: até 28 semanas e 6 dias e $\geq 29$ semanas

A análise estatística foi realizada pelo Software SPSS versão 22, com realização de avaliação descritiva dos dados (frequências absolutas e relativas das variáveis categóricas e medidas de tendência central e dispersão das quantitativas). O Teste Kolmogorv-Smirnov foi utilizado para verificar a distribuição normal das variáveis quantitativas. Na análise bivariada foram empregados os testes Qui-quadrado e t de Student e na multivariada a regressão logística binária. Para a análise múltipla optou-se para uso do modelo redu- zido (efeitos principais). Todas as variáveis com valores de "p" menores que 0,20 foram incluídas no primeiro modelo. Em seguida, foram retiradas as variáveis com valores de "p" maiores que 0,10 até que se chegasse ao modelo final. Foi considerado significante $p<$ 0,05 com intervalo de confiança de $95 \%$.

\section{RESULTADOS}

No período do estudo foram internados na UTIN 132 bebês de muito baixo peso e com idade gestacional $\leq 32$ semanas, sendo que destes, 9 tiveram que ser excluídos: 2 por malformações congênitas, 3 por recusa dos pais em participar do estudo e 4 por gemelaridade múltipla (quadrigêmeos), devido a possibilidade da ordem do nascimento dos bebês interferir na morbimortalidade. Sendo assim, 123 recém-nascidos foram incluídos na pesquisa, cujas características gerais estão listadas na Tabela 1.

Dos RNs incluídos, a média de peso ao nascer e idade gestacional ao nascimento foi respectivamente, $1079,2 \pm 270,8 \mathrm{~g}$ e 29 $\pm 2,6$ semanas. Foram diagnosticados com hemorragia pulmonar 37 bebês, totalizando $30,1 \%$ dos 123 bebês do estudo. Dentre os pacientes da amostra, ocorreu um maior número de bebês com peso $\geq 1000 \mathrm{~g}$ (76 bebês), dos quais 13,2\% apresentaram HP e 47 bebês com peso até $999 \mathrm{~g}$, sendo que destes, $27(57,4 \%)$ apresentaram HP. Com relação à idade gestacional, dos 123 bebês da amostra 59 nasceram com menos que 29 semanas, dos quais $28(47,5 \%)$ apresentaram HP e 64 nasceram com 29 semanas ou mais, dentre os quais, $9(14,1 \%)$ desenvolveram HP.

A associação entre a ocorrência de HP 
Tabela 1. Caracterização da amostra de recém-nascidos de muito baixo peso internados e idade gestacional $\leq 32$ semanas em Natal/RN

\begin{tabular}{|c|c|c|c|}
\hline \multicolumn{2}{|l|}{ Variáveis } & $n=123$ & Porcentagem \\
\hline \multirow{2}{*}{ Hipertensão gestacional } & Sim & 60 & 48,8 \\
\hline & Não & 63 & 51,2 \\
\hline \multirow{2}{*}{ Corticóide antenatal } & Não & 37 & 30 \\
\hline & Sim & 86 & 70 \\
\hline \multirow{2}{*}{ Idade gestacional (semanas) } & Até 28,6 & 59 & 48 \\
\hline & $\geq 29$ & 64 & 52 \\
\hline \multirow{2}{*}{ Tipo de Parto } & Normal & 45 & 36,6 \\
\hline & Cesárea & 78 & 63,4 \\
\hline \multirow{2}{*}{ Apgar no $5^{\circ}$ minuto } & $<7$ & 22 & 18 \\
\hline & $\geq 7$ & 100 & 82 \\
\hline \multirow{2}{*}{ Reanimação } & Sim & 75 & 61,5 \\
\hline & Não & 47 & 38,5 \\
\hline \multirow{2}{*}{ Peso de Nascimento (gramas) } & Até 999 & 47 & 38,2 \\
\hline & $\geq 1000$ & 76 & 61,8 \\
\hline \multirow{2}{*}{ Classificação peso ao nascer } & PIG & 25 & 20,3 \\
\hline & AIG & 98 & 79,7 \\
\hline \multirow{2}{*}{ Sexo } & Masculino & 70 & 57 \\
\hline & Feminino & 53 & 43 \\
\hline \multirow{2}{*}{ Sepse Precoce } & Sim & 65 & 52,8 \\
\hline & Não & 58 & 47,2 \\
\hline \multirow{2}{*}{ Presença de PCA } & Sim & 36 & 34,6 \\
\hline & Não & 68 & 65,4 \\
\hline \multirow{2}{*}{ Uso de Surfactante } & Sim & 78 & 63,4 \\
\hline & Não & 45 & 36,6 \\
\hline
\end{tabular}

PCA: persistência do canal arterial, PIG: pequeno para idade gestacional, AIG: adequado para idade gestacional.

e os fatores de risco elencados foi avaliada por meio de análise bivariada e do modelo de regressão logística multivariado. Na primeira análise, verificou-se uma associação positiva como fator de risco para HP as seguintes variáveis: menor IG e peso ao nascimento, presença de canal arterial patente, sepse precoce, ausência de uso de corticoide antenatal, necessidade de uso de surfactante e de manobras de reanimação neonatal em sala de parto e Índice de Apgar no $5^{\circ}$ minuto de vida $<7$.

Com relação às variáveis quantitativas, encontrou-se uma média de idade gestacional e de peso de nascimento dos 37 bebês que apresentaram HP de 27,2 $\pm 2,086$ semanas e $875,57 \pm 213,4 \mathrm{~g}$, respectivamente. A temperatura média de admissão na UTIN dos bebês que evoluíram com $\mathrm{HP}$ foi $34,2^{\circ} \mathrm{C} \pm 1,02$ versus $34,8^{\circ} \mathrm{C} \pm 1,14$ nos que não apresenta- 
Tabela 2. Análise de fatores de risco qualitativos com relação à ocorrência de hemorragia pulmonar em recém-nascidos de muito baixo peso e idade gestacional $\leq 32$ semanas admitidos em UTIN em Natal/RN

\begin{tabular}{|c|c|c|c|c|c|}
\hline \multirow{2}{*}{\multicolumn{2}{|c|}{ Variáveis }} & \multicolumn{2}{|c|}{$\begin{array}{l}\text { Hemorragia Pulmonar } \\
n=123\end{array}$} & \multirow{3}{*}{$\begin{array}{c}\begin{array}{c}\text { Valor de } \\
p\end{array} \\
0,708\end{array}$} & \multirow{3}{*}{$\frac{\text { OR (IC 95\%) }}{1,15(0,53-2,50)}$} \\
\hline & & $\operatorname{Sim} n(\%)$ & Não n (\%) & & \\
\hline $\begin{array}{l}\text { Hipertensão } \\
\text { gestacional }\end{array}$ & $\begin{array}{l}\text { Sim } \\
\text { Não }\end{array}$ & $\begin{array}{l}19(31,7) \\
18(28,6)\end{array}$ & $\begin{array}{l}41(68,3) \\
45(71,4)\end{array}$ & & \\
\hline $\begin{array}{l}\text { Corticóide } \\
\text { antenatal }\end{array}$ & $\begin{array}{l}\text { Não } \\
\text { Sim }\end{array}$ & $\begin{array}{l}19(51,4) \\
18(20,9)\end{array}$ & $\begin{array}{l}18(48,6) \\
68(79,1)\end{array}$ & 0,001 & $3,98(1,74-9,13)$ \\
\hline $\begin{array}{l}\text { Idade gestacional } \\
\text { (semanas) }^{1}\end{array}$ & $\begin{array}{c}\text { Até } 28,6 \\
\geq 29\end{array}$ & $\begin{array}{l}28(47,5) \\
09(14,1)\end{array}$ & $\begin{array}{l}31(52,5) \\
55(85,9)\end{array}$ & $<0,001$ & $3,37(1,74-6,54)$ \\
\hline Tipo de Parto & $\begin{array}{l}\text { Normal } \\
\text { Cesárea }\end{array}$ & $\begin{array}{l}14(31,1) \\
23(29,5)\end{array}$ & $\begin{array}{l}31(68,9) \\
55(70,5)\end{array}$ & 0,850 & $1,08(0,49-2,39)$ \\
\hline $\begin{array}{l}\text { Apgar no } 5^{\circ} \\
\text { minuto }^{1}\end{array}$ & $\begin{array}{l}<7 \\
\geq 7\end{array}$ & $\begin{array}{l}11(50) \\
25(25)\end{array}$ & $\begin{array}{l}11(50) \\
75(75)\end{array}$ & 0,02 & $3,01(1,16-7,76)$ \\
\hline $\begin{array}{l}\text { Reanimação } \\
\text { Neonatal }\end{array}$ & $\begin{array}{l}\text { Sim } \\
\text { Não }\end{array}$ & $\begin{array}{l}31(41,3) \\
06(12,8)\end{array}$ & $\begin{array}{l}44(58,7) \\
41(87,2)\end{array}$ & 0,001 & $4,81(1,82-12,73)$ \\
\hline $\begin{array}{l}\text { Peso de } \\
\text { Nascimento }(\mathrm{g})^{1}\end{array}$ & $\begin{array}{l}\text { Até } 999 \\
\geq 1000\end{array}$ & $\begin{array}{l}27(57,4) \\
10(13,2)\end{array}$ & $\begin{array}{l}20(42,6) \\
66(86,8)\end{array}$ & $<0,001$ & $8,91(3,69-21,51$ \\
\hline $\begin{array}{l}\text { Classificação } \\
\text { peso ao nascer }\end{array}$ & $\begin{array}{l}\text { PIG } \\
\text { AIG }\end{array}$ & $\begin{array}{l}07(28,0) \\
30(30,6)\end{array}$ & $\begin{array}{c}18(72) \\
68(69,4)\end{array}$ & 0,799 & $0,88(0,33-2,33)$ \\
\hline Sexo & $\begin{array}{l}\text { Masc } \\
\text { Fem }\end{array}$ & $\begin{array}{l}17(24,3) \\
20(37,7)\end{array}$ & $\begin{array}{l}53(75,7) \\
33(62,3)\end{array}$ & 0,107 & $1,21(0,95-1,56)$ \\
\hline Sepse Precoce & $\begin{array}{l}\text { Sim } \\
\text { Não }\end{array}$ & $\begin{array}{l}25(38,5) \\
12(20,7)\end{array}$ & $\begin{array}{l}40(61,5) \\
46(79,3)\end{array}$ & 0,032 & $2,39(1,07-5,37)$ \\
\hline PCA & $\begin{array}{l}\text { Sim } \\
\text { Não }\end{array}$ & $\begin{array}{c}21(58,3) \\
05(7,4)\end{array}$ & $\begin{array}{l}15(41,7) \\
63(92,6)\end{array}$ & $<0,001$ & $17,64(5,72-54,39)$ \\
\hline $\begin{array}{l}\text { Uso de } \\
\text { Surfactante }\end{array}$ & $\begin{array}{l}\text { Sim } \\
\text { Não }\end{array}$ & $\begin{array}{c}35(44,9) \\
02(4,4)\end{array}$ & $\begin{array}{l}43(55,1) \\
43(95,6)\end{array}$ & $<0,001$ & $17,5(3,96-77,36)$ \\
\hline HPIV & $\begin{array}{l}\text { Sim } \\
\text { Não }\end{array}$ & $\begin{array}{l}12(31,6) \\
15(22,4)\end{array}$ & $\begin{array}{l}26(68,4) \\
52(77,6)\end{array}$ & 0,30 & $1,60(0,65-3,90)$ \\
\hline
\end{tabular}

OR: odds-ratio, IC: intervalo de confiança, g: gramas, PCA: persistência do canal arterial, PIG: pequeno para idade gestacional, AIG: adequado para idade gestacional, HPIV: hemorragia peri-intraventricular. teste: Qui-quadrado.

ram a complicação estudada, diferença com significância estatística, apesar da média de temperatura baixa nos dois grupos, segundo dados na tabela a seguir.

$\mathrm{Na}$ análise multivariada apenas a ausência de uso de corticoide antenatal, menor peso de nascimento e persistência do canal arterial permaneceram associadas à hemorra- gia pulmonar. Sobre os pressupostos do modelo, o Omnibus Test que indica a significância do modelo, teve o valor de "p" significativo (< 0,001). O teste de Hosmer and Lemeshow utilizado para verificar o ajuste do modelo apresentou um valor de "p" não significativo $(0,254)$, o que indica um modelo ajustado. Em relação ao poder preditivo, a especificidade 
Tabela 3. Análise de fatores de risco quantitativos com relação a ocorrência ou não de hemorragia pulmonar em recém-nascidos de muito baixo peso e idade gestacional $\leq 32$ semanas admitidos em UTIN de Natal/RN

\begin{tabular}{|c|c|c|c|}
\hline \multirow[t]{2}{*}{ Fatores de Risco } & $\begin{array}{l}\text { Hemorragia } \\
\text { Pulmonar }\end{array}$ & Média (DP) & Valor de $\mathrm{P}$ \\
\hline & \multicolumn{3}{|c|}{$n=123$} \\
\hline \multirow[t]{2}{*}{ Idade Gestacional (semanas) } & Sim & $27,22(2,086)$ & \multirow[b]{2}{*}{$<0,001$} \\
\hline & Não & $29,29(2,165)$ & \\
\hline \multirow[t]{2}{*}{ Peso de Nascimento (gramas) } & Sim & $875,57(213,405)$ & \multirow{2}{*}{$<0,001$} \\
\hline & Não & $1147,13(250,069)$ & \\
\hline \multirow{2}{*}{$\begin{array}{l}\text { Temperatura de Admissão na UTIN } \\
\left({ }^{\circ} \mathrm{C}\right)\end{array}$} & Sim & $34,23(1,024)$ & \multirow[b]{2}{*}{0,007} \\
\hline & Não & $34,83(1,149)$ & \\
\hline
\end{tabular}

DP: desvio-padrão, UTIN: Unidade de terapia intensiva neonatal, ${ }^{\circ} \mathrm{C}$ : graus Celsius. Teste t de Student.

Tabela 4. Fatores de risco para hemorragia pulmonar em 123 recém-nascidos de muito baixo peso e idade gestacional $\leq 32$ semanas nascidos em Natal/RN (modelo final de regressão logística multivariada)

\begin{tabular}{|c|c|c|c|c|c|c|c|}
\hline \multirow{3}{*}{\multicolumn{2}{|c|}{ Variáveis }} & \multicolumn{6}{|c|}{ Hemorragia Pulmonar } \\
\hline & & \multicolumn{2}{|c|}{ Sim } & \multicolumn{2}{|c|}{ Não } & \multirow{4}{*}{$\frac{p}{0,001}$} & \multirow{4}{*}{$\begin{array}{c}\text { OR ajustado } \\
\text { (IC 95\%) } \\
8,54(2,44-29,94)\end{array}$} \\
\hline & & $n$ & $\%$ & $n$ & $\%$ & & \\
\hline \multirow[t]{2}{*}{ PCA } & Sim & 63 & 92,6 & 05 & 7,4 & & \\
\hline & Não & 15 & 41,7 & 21 & 58,3 & & \\
\hline \multirow{2}{*}{$\begin{array}{l}\text { Corticóide } \\
\text { antenatal }\end{array}$} & Fez uso & 68 & 79,1 & 18 & 20,9 & 0,011 & $6,08(1,52-24,37)$ \\
\hline & Não uso & 18 & 48,6 & 19 & 51,4 & 0,001 & $0,99(0,99-0,99)$ \\
\hline \multicolumn{2}{|c|}{$\begin{array}{l}\text { Peso ao Nascer- } \\
\text { gramas (média) }\end{array}$} & \multicolumn{2}{|c|}{1147,13} & \multicolumn{2}{|c|}{875,57} & 0,001 & $0,99(0,99-0,99)$ \\
\hline
\end{tabular}

OR: odds-ratio, IC: intervalo de confiança, PCA: persistência do canal arterial $-0,005$ PN + 2,145 PCA + 1,806 Corticóide antenatal

encontrada foi de $92,3 \%$ e a sensibilidade de $76,9 \%$, com porcentagem geral de acerto de $88,5 \%$.

A análise também mostrou que para cada grama adicional no $\mathrm{PN}$ diminui em $0,5 \%$ a chance de desenvolver HP. O RN com PCA e aquele cuja mãe não fez uso de corticoide antenatal teve 8,54 vezes e 6,08 vezes mais chance, respectivamente, de apresentar hemorragia pulmonar.

\section{DISCUSSÃO}

A incidência de HP encontrada foi elevada comparada com outros estudos, porém a amostra foi formada por bebês, sob maior risco de HP por serem todos prematuros $\leq 32$ semanas e com peso de nascimento menor que $1500 \mathrm{~g}$. Os dados na literatura com relação a incidência também são bastante variados, 
visto que a própria definição de HP se altera entre eles (desde achados puramente clínicos até a necessidade de queda de hematócrito ou presença de imagem em RX de tórax). Além disso, em sua maioria, são estudos pequenos, realizados em apenas um centro, com um número reduzido de pacientes, assim como neste trabalho. Apenas na pesquisa de Ahmad et al. ${ }^{4}$, realizada com 2799 recém-nascidos diagnosticados com HP, encontra-se dados de vários centros de pesquisa com um número mais significativo de pacientes, no qual a incidência de HP variou amplamente entre prematuros extremos e aqueles nascidos com idades gestacionais maiores (de $86 \%$ de incidência com 23 semanas de idade gestacional até uma redução para 0,6\% em nascidos maiores que 32 semanas de idade gestacional).

Neste estudo a estratificação de acordo com a idade gestacional foi realizada em apenas dois grupos, nascidos até 28 semanas e 6 dias e $\geq 29$ semanas. A incidência de HP foi de $47,5 \%$ e $14,1 \%$ no primeiro e segundo grupos, respectivamente. Uma importante redução na ocorrência de HP com o aumento da IG, informação confirmada pela análise da idade gestacional no formato quantitativo, tornando a maior prematuridade um fator de risco significativo estatisticamente na avaliação bivariada $(p<0,001)$, dado compatível com diversos estudos na literatura ${ }^{1,3,4,11}$. Entretanto, essa associação não se manteve no modelo de regressão logística multivariado.

Assim como neste trabalho, vários estudos na literatura observaram uma associa- ção de uso de surfactante após o nascimento com ocorrência de $\mathrm{HP}^{3,10,14}$. A administração de surfactante em prematuros com canal arterial poderia estar ligada a uma melhora da complacência pulmonar, com diminuição do colabamento alveolar e, por conseguinte, uma redução na pressão e resistência pulmonar, levando a um aumento do fluxo sanguíneo pelo canal arterial, como demonstram os estudos de Scholl ${ }^{2}$ e Chen ${ }^{3}$. Além disso, o surfactante melhora a função pulmonar e a não redução dos parâmetros ventilatórios após sua administração poderia também causar lesão pulmonar e aumentar os riscos de HP. No estudo atual, entretanto, outras variáveis foram mais importantes, de tal forma que no modelo de regressão logística multivariado, o uso de surfactante como fator de risco para HP não se manteve.

A associação de HP com a sepse precoce foi positiva na análise bivariada, porém a associação também não se manteve na análise multivariada. Segundo o estudo de Wang ${ }^{21}$, a sepse precoce foi um fator de risco independente para HP, podendo elevar em 3,4 vezes a chance deste desfecho. A presença de infecção aumentaria a permeabilidade vascular pulmonar, elevando o risco de desenvolvimento de $\mathrm{HP}^{3}$, porém, outros fatores que não foram avaliados nesse estudo poderiam interferir nessa análise, tendo em vista que muitas vezes o recém-nascido com sepse também está em ventilação mecânica agressiva, recebendo expansões, fazendo uso de drogas vasoativas por choque séptico, apresentando trombocitopenia decorrente da 
sepse, dentre outros fatores, que também poderiam contribuir para o sangramento pulmonar.

A necessidade de reanimação em sala de parto também foi um fator de risco encontrado para HP na análise bivariada. Entretanto, não foi realizada uma avaliação especifica sobre até qual etapa da reanimação foi necessário chegar nestes bebês que evoluíram com HP posteriormente, não sendo possível afirmar qual exatamente pode ter sido o fator de risco associado (ventilação com pressão positiva com máscara, intubação em sala de parto, necessidade de adrenalina ou expansão para reanimação cardiopulmonar). A necessidade de ventilação positiva poderia ser um fator associado na medida em que causa distensão alveolar e possivelmente lesão de capilares pulmonares, aumentando a fragilidade pulmonar ao sangramento ${ }^{1,11}$. Nos estudos de Ferreira e Carmona ${ }^{1}$ e Berger ${ }^{11}$, a intubação em sala de parto foi vista como fator de risco para HP.

O índice de Apgar $<7$ apresentou-se como um fator de risco para HP, porém, pode-se dizer que também está diretamente ligado à necessidade de reanimação em sala de parto. A asfixia neonatal pode cursar com trombocitopenia ${ }^{3}$ e/ou disfunção ventricular esquerda ${ }^{22}$, sendo essas as alterações que possivelmente contribuiriam para ocorrência da HP. No estudo de Chen ${ }^{3}$ também foi vista uma associação com asfixia neonatal e maior probabilidade de evolução para HP.

Após a análise com modelo de regressão logística multivariada, observou-se que permaneceram relevantes como fatores de risco para HP apenas a presença de canal arterial patente, ausência de uso de corticoide antenatal e menor peso de nascimento.

A média de peso entre os bebês que apresentaram HP foi de $875,5 \mathrm{~g}$ e $1147,1 \mathrm{~g}$ naqueles que não apresentaram, sendo que dos 37 bebês com HP, 27 (73\%) pesaram até $999 \mathrm{~g}$. Resultado semelhante foi encontrado em diversos estudos da literatura ${ }^{1,3,4}$, corroborando para a hipótese de que a HP é mais prevalente quanto menor o peso de nascimento. No estudo brasileiro de Ferreira e Carmona ${ }^{1}, 92,4 \%$ dos bebês que apresentaram HP nasceram com peso inferior a $1500 \mathrm{~g}$, e no estudo de $\mathrm{Chen}^{3}$, o peso médio dos bebês com HP maciça foi de $864,9 \mathrm{~g}+/-301,4 \mathrm{~g}$, se assemelhando aos dados desse estudo.

Outro fator importante com forte associação para ocorrência de HP encontrado nesse trabalho foi a presença de canal arterial patente após $72 \mathrm{~h}$ de nascimento, que promove fluxo entre a artéria pulmonar e aorta. Após o nascimento, com a queda da pressão pulmonar, o fluxo pelo canal tende a ser da aorta para a artéria pulmonar (shunt esquerdo-direito), aumentando o fluxo sanguíneo pulmonar e, como consequência, aumentando a possibilidade de ocorrência de HP em prematuros ${ }^{23}$. Nesse estudo, nem todos os bebês tiveram avaliação ecocardiográfica (104 dos 123 bebês foram avaliados), ainda assim observou-se uma associação positiva no modelo de regressão logística multivariado com um odds ratio de $8,54(p<0,001)$, sendo um resultado compatível com vários publicados na literatura ${ }^{2,4-9,23}$. A pesquisa de Ahmad et al. ${ }^{4}$ também avaliou a presença de 
canal arterial patente, observando uma menor exposição a indometacina profilática entre os pacientes que evoluíram com HP. Apesar da associação entre a PCA e a HP, o fechamento medicamentoso ou cirúrgico do canal arterial é um tema controverso na literatura, com alguns estudos ainda em andamento.

Com relação ao uso de corticoide antenatal, os resultados foram semelhantes aos encontrados por Berger et al. ${ }^{11}$, sendo um fator protetor para a ocorrência de HP. O uso de corticoide ajuda na maturação pulmonar, o que também está associado com menores chances de uso de surfactante e necessidade de ventilação mecânica, reduzindo também a mortalidade em prematuros ${ }^{12}$. Quando colocado no modelo de regressão logística multivariado, o não uso de corticoide antenatal permaneceu como fator importante para o desenvolvimento de HP com odds ratio de 6,08.

Dessa forma, os resultados dessa pesquisa foram compatíveis com o que existe na literatura atualmente. É possível que o número reduzido de pacientes tenha sido um fator limitante para a análise. Além disso, existem outros fatores que podem estar associados a ocorrência de HP que não foram avaliados como: dados mais precisos do tipo de suporte ventilatório na época em que ocorreu o sangramento pulmonar, uso de hemocomponentes ou expansões prévias à hemorragia, uso de drogas vasoativas, valores de plaquetas, coagulograma e até mesmo dados de gasometria, visto que os valores de $\mathrm{pH}$ e $\mathrm{pCO} 2$ poderiam interferir na vasodilatação pulmonar. Porém, também são escassos os dados na literatura para verificar tais associações. Por outro lado, a metodologia empregada nesse estudo foi prospectiva, conferindo maior confiabilidade das informações colhidas.

\section{CONCLUSÃO}

Nesse estudo observou-se uma alta incidência de HP em recém-nascidos de muito baixo peso e $\leq 32$ semanas, bem como uma associação dessa complicação com menor peso de nascimento, presença de canal arterial após $72 \mathrm{~h}$ de vida e não uso de corticoide antenatal. Todos esses fatores de risco podem ser modificados com uma melhor assistência à mãe durante o seu pré-natal (prevenção de prematuridade extrema e nascimentos de recém-nascidos de muito baixo peso e administração de corticoide antenatal quando o parto prematuro $<34$ semanas é previsto para os próximos 7 dias) e ao recém-nascido prematuro após o nascimento (ações para prevenção PCA). Tais medidas, podem contribuir para uma redução da incidência dessa importante morbidade e causa de óbito nessa população. 


\section{REFERÊNCIAS}

1. Ferreira $\mathrm{CH}$, Carmona F, Martinez FE. Prevalence, risk factors and outcomes associated with pulmonary hemorrhage in newborns. J Pediatr. 2014; 90:316-22.

2. Scholl JE, Yanowitz TD. Pulmonary hemorrhage in very low birth weight infants: a case-control analysis. J Pediatr. 2015; 166:1083-1084.

3. Chen YY, Wang HP, Lin SM, Chang JT, Hsieh KS, Huang FK, et al. Pulmonary hemorrhage in very low-birthweight infants: risk factors and management. Pediatr Int. 2012; 54:743-747.

4. Ahmad KA, Bennett MM, Ahmad SF, Clark RH, Tolia VN. Morbidity and mortality with early pulmonary haemorrhage in preterm neonates. Arch Dis Child Fetal Neonatal Ed. 2018; 0:F1-F6.

5. Garland J, Buck R,Weinberg M. Pulmonary hemorrhage risk in infants with a clinically diagnosed patent ductus arteriosus: A retrospective cohort study.Pediatrics.1994; 94:719-723.

6. Kluckow M, Evans N. Ductal shunting, high pulmonary blood flow, and pulmonary hemorrhage. J Pediatr. 2000; 137:68-72.

7. William E. Benitz and Committee on fetus and newborn Patent Ductus Arteriosus in Preterm Infants. Pediatrics. 2016, 137 (1). e20153730.

8. Rozé J, Cambonie G, Marchand-Martin L, Gournay V, Durrmeyer X, Durox M, et al.Association Between Early Screening for Patent Ductus Arteriosus and In Hospital Mortality Among Extremely Preterm Infants. JAMA. 2015; 313(24): 2441-2448.

9. Su BH, Lin HY,Huang FK, Tsai ML,Huang YT. Circulatory Management Focus ing on Preventing Intraventricular Hemorrhage and Pulmonary Hemorrhage in Preterm Infants. Pediatrics and Neonatology. 2016; 57,453-462.

10. Raju TN, Langenberg P. Pulmonary hemorrhage and exogenous surfactant therapy: a metaanalysis. J Pediatr. 1993; 123:603-610.

11. Berger, T, Allred, E, Van MarterL. Antecedents of Clinically Significant Pulmonary Hemorrhage Among New Born Infants. J Perinatol. 2000; 20:295-300.
12. Travers CP, Carlo WA, McDonald SA, Newman NS, Higgins RD. Mortality and pulmonary outcomes of extremely preterm infants exposed to antenatal corticosteroids. Am J Obstet Gynecol. 2018; (218):130:1-13.

13. Tsou KI, The Taiwan Premature Infant Developmental Collaborative Study Group. The morbidity and survival of very low birth weight infants in Taiwan: Changes in 10 years. In: White paper. Premature Baby Foundation of Taiwan, Taipei. 2011; 5-24.

14. Pandit PB, O'Brien K, Asztalos E, Colucci E, Dunn MS. Outcome following pulmonary haemorrhage in very low birthweight neonates treated with surfactant. Arch Dis Child Fetal Neonatal Ed. 1999; 81:40-44.

15. Lee NY, Lee HY, Park SH, Sung IK, Youn YA. Massive pulmonary hemorrhage and severe retinopathy of prematurity in very low birth weight infants. Turk J Pediatr. 2017; 59:636-641.

16. Lee M, Wu K, Yu A, Roumiantsev S, Shailam R, Nimkin $\mathrm{K}$, et al. Pulmonary hemorrhage in neonatal respiratory distress syndrome: Radiographic evolution, course, complications and long-term clinical outcomes. J Neonatal Perinatal Med. 2019; 12(2):161-171.

17. Brasil. Agência Nacional de Vigilância Sanitária. Critérios Diagnósticos de Infecções Relacionadas à Assistência à Saúde/Agência Nacional de Vigilância Sanitária. Brasília: Anvisa, 2017.

18. Villar J, Ismail LC, Victora CG, Ohuma EO. The International Fetal and Newborn Growth Consortium for the 21st Century (INTERGROWTH-21st). International standards for newborn weight, length, and head circumference by gestational age and sex: the Newborn Cross-Sectional Study from the INTERGROWTH-21st Project. Lancet. 2014; 384:857-868.

19. Villar J, Giuliani F, Fenton TR, Ohuma EO, Ismail LC, Kennedy $\mathrm{SH}$ et al. Intergrow-21st very preterm size at birth reference charts. Lancet. 2016; 387(10021):844-845.

20. Papile LA, Burstein J, Burstein R, Koffler H. Incidence and Evolution of subependymal and intraventricular hemorrhage: a study of infants with birthweights less than 1,500 g. J Pediatr. 1978; 92(4):529-534. 
21. Wang $\mathrm{T}$, Zhou M, Hu X, Liu J .Perinatal risk factors for pulmonary hemorrhage in extremely low-birth-weight infants. World J Pediatr. 2020; (16):299-304.

22. Gillam-Krakauer M, Gowen Jr CW. Birth Asphyxia. [Updated 2020 Apr 23]. In: StatPearls [Internet]. Treasure Island (FL): StatPearls Publishing; 2020.

23. Kluckow M, Evans N. Ductal shunting, high pulmonary blood flow, and pulmonary hemorrhage. J Pediatr. 2000; 137:68-72.

\section{CORRESPONDÊNCIA}

Cintia Suemy Uehara

Rua Dr. Ewerton Dantas Cortes, 1504 - apto 502

Condomínio Nova Amsterdam/ Tirol Natal-RN / CEP 59020-620

E-mail: cintiamemi@yahoo.com.br 